\title{
Physical Nature of Length Contraction (Part 2) How the Electron Elongates While Its Orbit Contracts
}

\author{
Conrad Ranzan \\ Astrophysics Department, DSSU Research, Niagara Falls, Ontario, Canada
}

\section{Email address:}

ranzan@cellularuniverse.org

\section{To cite this article:}

Conrad Ranzan. Physical Nature of Length Contraction (Part 2) How the Electron Elongates While Its Orbit Contracts. International Journal of High Energy Physics. Vol. 6, No. 1, 2019, pp. 1-12. doi: 10.11648/j.ijhep.20190601.11

Received: March 23, 2019; Accepted: April 30, 2019; Published: May 23, 2019

\begin{abstract}
The recent discovery of the process/mechanism of the total conversion of mass to energy -a process that does not involve matter-antimatter interaction - has radically altered the theory of physical length contraction. It is argued that while atoms and molecules (and therefore also objects) undergo length contraction during aether-referenced motion the constituent fundamental particles themselves, on the other hand, do not! Fundamental particles actually undergo a strictly limited elongation. Based on the sound evidence and supporting theory that all elementary particles are configurations of photons, four supporting arguments are presented: (i) Particle elongation facilitates the conservation of its total intrinsic energy. (ii) The decrease of mass (as defined) becomes necessary with increased motion (with respect to aether). (iii) Elongation provides the key element in the mechanism of mass-to-energy conversion. A noninteraction-hundred-percent conversion (i.e., no particle-antiparticle annihilations is involved). (iv) The preclusion of the formation of singularity-type black holes, thus avoiding the associated infinity problems. Under the traditional view one cannot combine unrestricted length contraction and energy conservation - not in a realistic way. And for this very reason the long-standing rule has been that a mass particle/object can never attain lightspeed. And that rule, in turn, has led to a failure to understand the nature of the strangest objects of the Universe - Terminal neutron stars. The new interpretation overcomes this difficulty. The essential point being advanced is that Nature has a way for the total conversion of mass to energy. It accomplishes this by changing the gravitational environment — by subjecting "stationary" mass to lightspeed aether flow, compelling mass to undergo conversion, which requires length elongation.
\end{abstract}

Keywords: Electron Model, Electron Propagation, Nonmaterial Aether, Relativistic Mass, Intrinsic Mass and Energy, Mass-Energy Conversion, Terminal Neutron Star, DSSU Theory

\section{Introducing the Electron}

For a proper understanding of relativistic length contraction, the nature of the electron is of foremost importance.

The electron is the carrier of the fundamental electric charge and was for many years considered to be a point particle. It acted like a point charge; it could not be divided into smaller units; and it did not seem to have any subcomponents. And so, based on the available evidence theorists were led to the belief in a structureless electron. However, the point-charge idea leads to a rather obvious problem - the zero-radius problem. The issue of the electron without a radius was a challenging problem of modern theoretical physics. "A point-like electron (zero radius) generates serious mathematical difficulties due to the self-energy of the electron tending to infinity." [1] On the other hand, the admission of the hypothesis of a finite radius of the electron was said to be incompatible with the premises of the theory of relativity. However, since that objection was based on an incomplete theory of relativity (incomplete because it ignores the universal space medium, the one special reference frame), it had dubious validity. This left the door open to alternate ideas. There is, thus, no theoretical barrier to hypothesizing structure. If there is to be a deeper understanding of the electron, there has to be some sort of structure.

The structure of the electron is now believed to be a closed two-turn helix that is generated by the circular motion of a massless particle traveling at the speed of light [2]. Furthermore, by its looping motion it somehow projects an electromagnetic field - a negative charge. It is quite easy to 
determine the identity of the particle. There are only two kinds of particles that are massless and travel at lightspeed: photons and neutrinos. Neutrinos may immediately be disqualified since they display no external electromagnetic field (which is the very reason they are virtually undetectable). Photons, however, do. Such an external electric field is essential for manifesting an electric charge. If a self-looping photon is polarized in such a way so that the peak electric lines of force are always directed radially outward then what you have is essentially a charge-generating structural particle. And by convention, these radial force lines are pointing inward in the case of electrons (and outward for antielectrons).

A photon, when appropriately circularly polarized and confined, will manifest an electric charge. The nature of the confinement is shown in Figure 1.

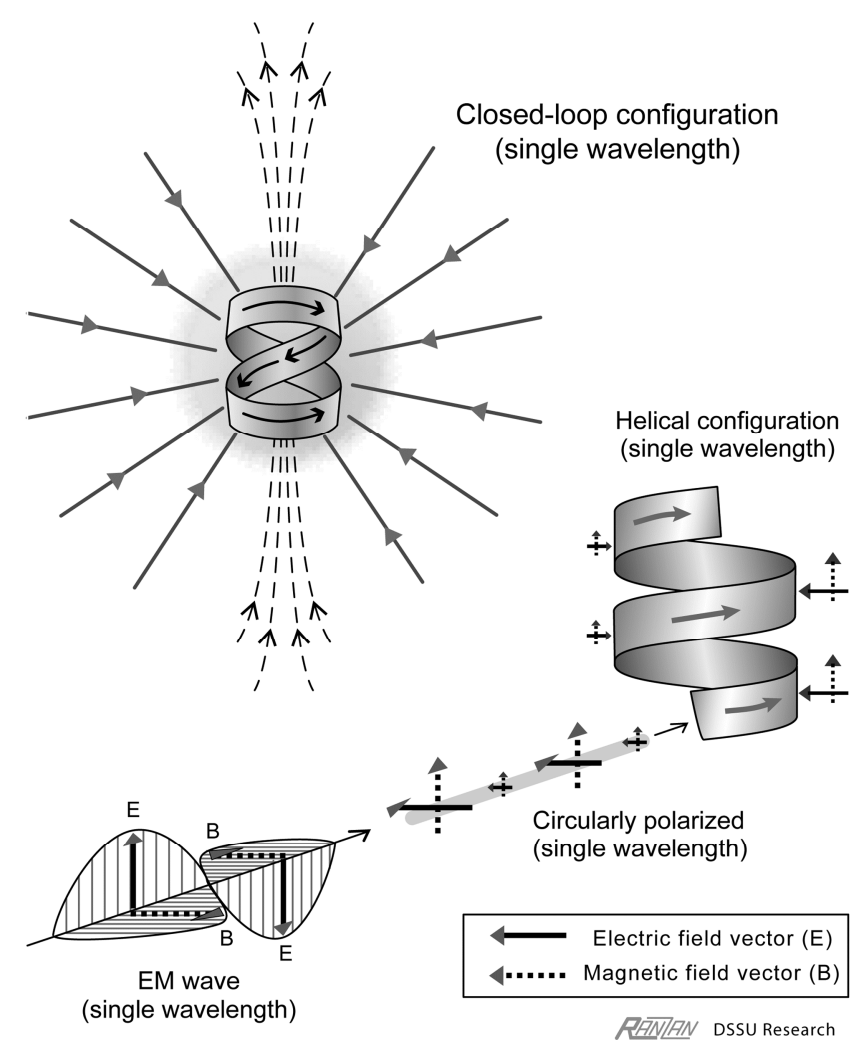

Figure 1. A high-energy photon, as represented here by the single wavelength electromagnetic pulse, when circularly polarized and spatially confined, transforms into an electron. The rotation of the polarization is such that the magnetic field vectors are in the plane of the helical strip and the electric field vectors are perpendicular to it. The electric field produces the charge monopole (negative); and the magnetic field produces the magnetic dipole. The electron is essentially a self-looping single wavelength photon with its electric field vectors always directed inward. (The antielectron, the positron, differs only in that its electric field vectors are directed outward.) This is by far the simplest model of the electron, while at the same time retaining consistency with reality.

Confinement - the state of a photon being spatially trapped - is significant for another reason, beyond the generation of charge. It is the defining attribute of mass. It is the reason that a confined photon represents mass while the photon itself is entirely massless. A general rule of confinement extends to other subatomic particles. The greater the photon confinement, the greater is its manifestation of the mass property. A nucleon, such as a neutron or a proton, manifests a greater mass simply because its constituent photons (it is probably a configuration of more than one) are confined within a smaller volume than is the confinement found in electrons. The greater degree of confinement is naturally related to the shorter wavelength of the more tightly looping photons. The point is that the "confinement" provides the electron with its charge and its mass.

The electron spin property, the electron's angular moment, fits nicely into this model. The self-orbiting motion of the photon is the source of the rotational moment. The model also accounts for the source of the electron's magnetic dipole (Figure 1). The electron's Compton wavelength also fits into the scheme. The wavelength of the self-looping photon is a key characteristic of electron; it defines the size of the particle and is known as the Compton wavelength. (The Compton wavelength of an electron can easily be computed, being, as it is, equal to Planck's constant divided by the product of the electron's mass and the speed of light. It is found to be $2.4 \times 10^{-12}$ meter.)

The investigations of Williamson [3], Qiu-Hong $\mathrm{Hu}$ [2], and others have convincingly shown that the electron is essentially a self-looping photon -a photon whose polarization orients the electric field radially and the magnetic field axially. Furthermore, as already noted, the wavelength of the electron's constituent photon corresponds to its Compton wavelength. The Williamson electron is the simplest - and yet most problem-free - model of the electron that has ever been devised.

Remarkably, the photon, possessing no inertial mass and no electric charge, is able to manifest a configuration exhibiting both. This is the electron; it will serve as the main player in this article.

\section{The Nature of Photon Confinement}

The nature of the loop constituting the electron is critically important for determining the electron's change-of-length characteristics. Incidentally, one might be tempted to think that a point-like electron solves the question of relativistic change-of-length; for a point, there is no length to change. However, one would be wrong; there would still be the electron's electric charge to consider - an external sphere of influence with inverse-square characteristics. It turns out, the loop configuration simplifies the analysis.

The electron is a single wavelength photon configured as a loop. The question is, is it a seamless closed loop, in the sense of the wave being seamless and continuous? Or does the looping photon have a front end and a back end? It is an important question. The nature of the electron's behavior when undergoing relativistic motion depends on this issue. Whether or not the electron undergoes contraction depends on the loop's continuous or discontinuous property.

The importance of photon confinement extends beyond just the electron. Within the developing theory of everything it applies to all subatomic particle —all mass-manifesting 
fundamental particles.

Just for the sake of the discussion, the photonic loop is assumed to be closed. Moreover, the loop is assumed to remain intact regardless of the electron's intrinsic motion (motion with respect to the universal space medium). It is then possible to make the following straightforward argument.

Consider the circular photon loop shown in Figure 2a. The time for some arbitrary point on the photon's wave to circumnavigate the loop is, say, $\Delta t_{0}$. The point starts at the lower part of the $y$-axis, makes one orbit, ending back at the $y$-axis starting location. Associated with this circular trajectory is a self-evident fact: The round trip time of the "point's" motion parallel to the horizontal $x$-axis must be equal to the round trip time of the motion parallel to the vertical $y$-axis. Both must be equal to $\Delta t_{0}$.

Next, the orbital path is projected onto the two axes as shown in Figure $2 \mathrm{~b}$ and $2 \mathrm{c}$. Imagine the photon propagating along these new paths. (The photon still propagates at the speed of light.) The paths are obviously shorter; therefore, the round trip times will be less than the original $\Delta t_{0}$. However, because of the symmetry (rotational symmetry) of the original loop, the round trip periods are shortened by the same fractional amount. Thus, for the $x$-axis round trip, $\Delta t_{\mathrm{x}}$ equals $F \Delta t_{0}$; and for the $y$-axis round trip, $\Delta t_{\mathrm{y}}$ equals $F \Delta t_{0}$. And so,

$$
\Delta t_{\mathrm{x}}=\Delta t_{\mathrm{y}} .
$$

(a)
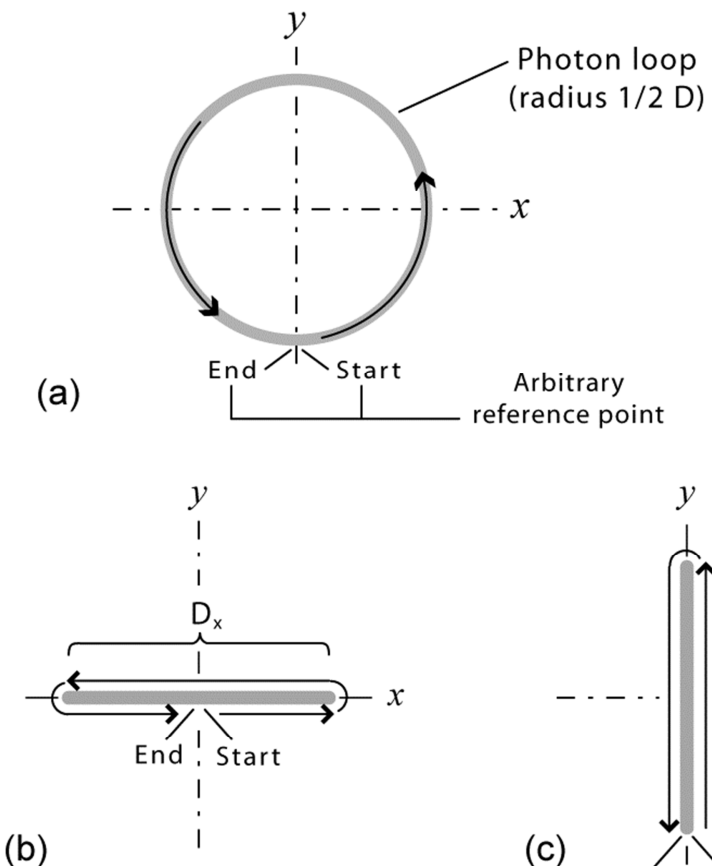

(c)

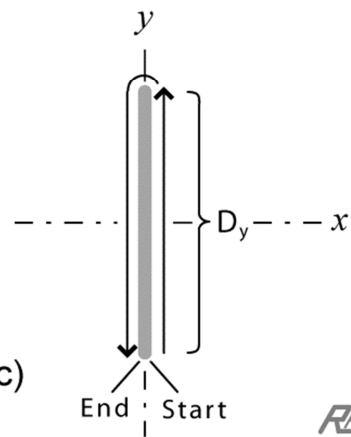

Figure 2. Equal time-of-travel argument for cross-direction circuit AND in-line-direction circuit. Part (a) shows the schematic of an electron with negligible intrinsic motion. The electron's "confined" photon propagates $C C W$ with orbital period of $\Delta t_{0}$. Part (b), the orbital path is shown projected onto the x-axis. This

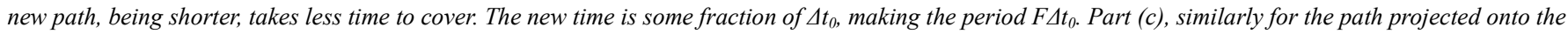
$y$-axis, a round trip is a fraction of $\Delta t_{0}$. Because of the symmetry involved, the time fraction must be the same. So the period is again $F \Delta t_{0}$.

Now imagine, if you will, traveling with the electron as it moves through the universal space medium (but without yourself being affected by the motion in any way). The electron and you, the unaffected observer, are moving in the positive $y$-axis direction. Another, even simpler way, to think of this is to treat the electron as being stationary while the universal space medium is flowing. The space medium is then pictured as streaming in the negative $y$-axis direction.

With the latter perspective in mind, the motion of the electron's constituent photon along the projected paths will now be analyzed. The analysis of these paths is simply a matter of working with velocity-vector triangles. For the intrinsic velocity along the $x$-axis path, as derived in Figure 3,

$$
\left|v_{x}\right|=\sqrt{c^{2}-v_{\mathrm{a}}^{2}} .
$$

The propagation time is then simply the lengths of the three path segments divided by the velocity magnitude,

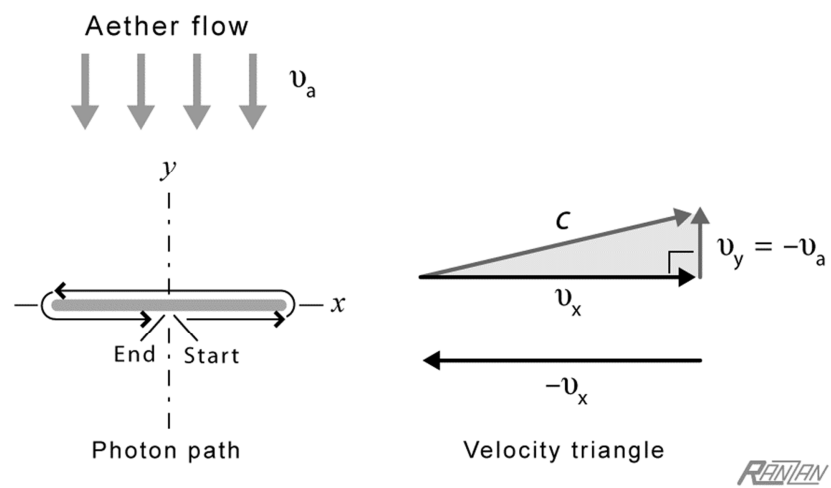

Figure 3. Motion of the photon along the $x$-axis circuit. Vector $v_{x}$ is the photon's velocity with respect to the coordinate system. Vector $c$ is the photon's velocity with respect to the luminiferous aether. Vector $v_{a}$ is the velocity of the aether. According to the velocity triangle, $\left|v_{x}\right|=\left(c^{2}-v_{a}^{2}\right)^{1 / 2}$. 
Next, consider the $y$-axis circuit.

As derived in Figure 4, the intrinsic velocity along the positive direction of the $y$-axis is

$$
\left|v_{y+}\right|=c-v_{\mathrm{a}} ;
$$

and along the negative direction of the $y$-axis it is,

$$
\left|v_{y-}\right|=c+v_{\mathrm{a}} .
$$

The propagation time is then simply the lengths of the two path segments divided by the respective velocity magnitudes,

$$
\Delta t_{y}=\frac{D_{y}}{c-v_{\mathrm{a}}}+\frac{D_{y}}{c+v_{\mathrm{a}}} .
$$

It was already determined that $\Delta t_{\mathrm{x}}$ equals $\Delta t_{\mathrm{y}}$. Therefore equations (3) and (6) can be combined as follows:

$$
\frac{2 D_{x}}{\sqrt{c^{2}-v_{\mathrm{a}}^{2}}}=\frac{D_{y}}{c-v_{\mathrm{a}}}+\frac{D_{y}}{c+v_{\mathrm{a}}},
$$

which simplifies to

$$
\frac{D_{y}}{D_{x}}=\sqrt{1-\left(v_{\mathrm{a}} / c\right)^{2}} .
$$

Expressed another way, in terms of the conventional symbolism

$$
\frac{D_{y}}{D_{x}}=\frac{1}{\gamma} .
$$

This is similar to the classic expression for length contraction in which $\gamma$ is the Lorentz gamma factor. (Gamma, $\gamma$, equals $1 / \sqrt{1-\left(v_{\mathrm{a}} / c\right)^{2}}$ and, of course, varies with the particle's speed.) The difference is that here $\gamma$ serves as an intrinsic gamma factor, which varies with aether-referenced motion (the intrinsic speed); while conventionally, the Lorentzian $\gamma$ serves as a relative gamma factor, which varies with arbitrarily chosen relative motion.

Since the gamma factor can only have a value of 1 or greater than 1 , it follows from equation (9) that

$$
\frac{D_{y}}{D_{x}}=\frac{1}{\gamma} \leq 1 \text { and } D_{y} \leq D_{x} .
$$

In other words, the above argument predicts that the diameter $D_{y}$ of the electron's photon loop shrinks. All $y$-dimensions would become smaller as the velocity $v_{a}$ increases. Based on the assumption of a closed photonic loop, the electron becomes flattened in the direction of intrinsic motion.
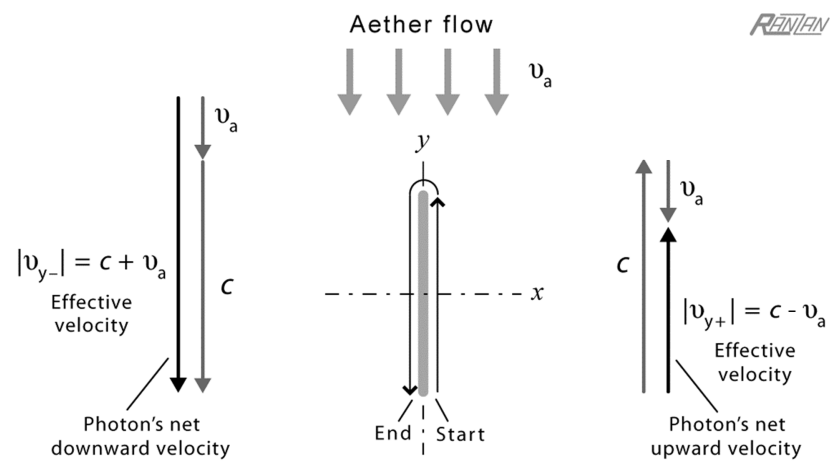

Figure 4. Motion of the photon along the y-axis circuit. Vector $v_{y}$ is the photon's velocity with respect to the coordinate system. Vector $c$ is the photon's velocity with respect to the luminiferous aether. Vector $v_{a}$ is the velocity of the aether. The photon's net velocity is $\left(c-v_{a}\right)$ during the upward portion of the path and $\left(c+v_{a}\right)$ during the downward portion of the path.

If the electron propagates as a photonic closed loop, if the loop is assumed to be continuous and unbroken, then length contraction (in direction of motion) must occur!

However, there are powerful arguments against the concept of unconditional loop closure.

\section{Three Arguments Against Closed Loop}

Although the electron propagates as a self-orbiting photon, the path of the orbit is not a strictly contiguous loop. There are three compelling arguments against the seamless-closed-loop concept.

\subsection{Untenable Implication for Mass Definition}

As was proven above, the insistence of loop closure causes the contraction of the photon loop - namely, a foreshortening of the loop in the direction of motion. What this means is that the photon becomes more confined. In the case of an electron, the photonic double-loop would become smaller (as shown in Figure 5). (Incidentally, the orientation of the closed loop does not alter the contraction/flattening; loop closure and length contraction are two sides of the same coin. They always go together.) It is a fundamental rule of elementary particles: the degree of confinement determines the mass attribute of any particle. A length contracted electron represents a tighter confinement; therefore, if this were to actually occur, it would represent an electron of greater mass - greater than the mass of an at-rest electron.

However, such increase in mass with increased motion applies only for the unrealistic assumption of closed-loop propagation. There is simply no evidence for mass gain - not theoretical and not experiential. The original theoretical notion of relativistic mass gain was abandoned, even by Einstein himself, by the middle of the $20^{\text {th }}$ century [4]. 


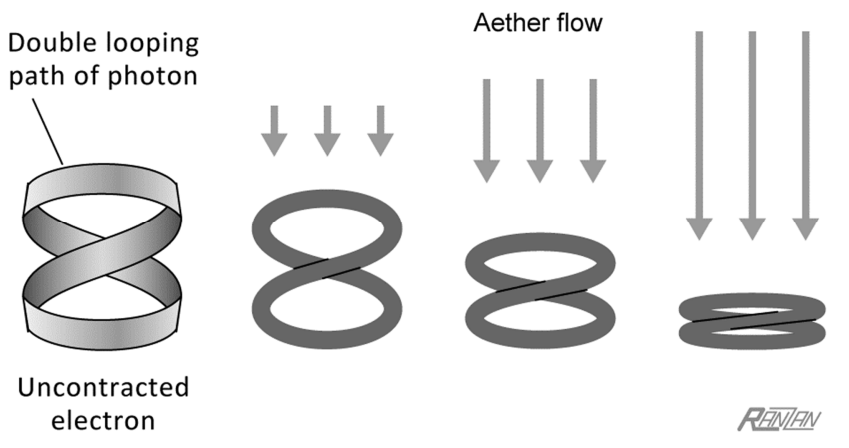

Figure 5. The assumption of loop closure brings with it the phenomenon of intrinsic length contraction. This means the self-looping photon, when subjected to increased aether flow, becomes ever more confined into a smaller region. By definition, the tighter the confinement, the greater is the particle's mass. The problem is that mass particles do not actually increase their mass value when there is an increase in motion. The original loop-closure assumption leads to a contradiction.

In a nutshell then: The seamless-closed-loop concept implies length contraction. Length contraction means greater photon confinement. Greater confinement, in turn, implies an increase of mass, for which there is no supporting evidence.

\subsection{Adverse Implication for Energy Conservation}

Another unambiguous argument is made here. The seamless-closed-loop concept with its attendant length contraction has the effect of shortening the path length. Of course, the loop's path length and the photon's wavelength are one and the same. One defines the other. And so, any change in the path length represents an explicit change in the photon's energy. (Keep in mind, this is being considered from the frame of the electron itself and independent of any observer.) A shorter path length represents a photon of higher energy.

In other words, by increasing the aether flow to the electron, a gain in energy is implied. This would represent a violation of the principle of conservation of intrinsic energy.

This is particularly troubling since no energy is being added to the electron. No force is being applied. There should not be any change in the photon's wavelength. The electron is not being accelerated by some electromagnetic field. (Only the gravitational environment is being altered, as will be demonstrated in the next subsection. The electron, or any other subatomic particle one may choose to consider, is merely "resting" on the surface of a body with an intense gravity field.)

Moreover, by confining the discussion to particles that are at rest with respect to some gravitating body, there is then no need to deal with kinetic energy.

\subsection{Adverse Implication for Mass-to-Energy Conversion}

The key point here is that the seamless-closed-loop concept would preclude the conversion of a mass particle (the electron in this case) into pure energy. There would simply be no way to unconfine the confined photon, except, of course, by particle-antiparticle annihilation. Stated another way, there would be no way to bring the electron up to the speed of light.

Within standard physics this contingency is not considered a problem. The academic physics community, because it is still lacking a complete theory of gravity, simply accepts as normal the theoretical impossibility for an electron to attain the speed of light.

However, if contrary to the conventional wisdom, an electron were somehow brought to the ultimate speed, something remarkable happens. It transforms to pure energy; it transforms into its massless form; its previously confined photon becomes a linearly propagating photon. As described by the eminent physicist John A. Wheeler,

"in this extreme relativistic limit a particle of rest mass $m$ behaves - so far as concerns the laws of conservation of momentum and energy - in practically the same way as a photon." [5]

Why is it so important to facilitate the attainment of light speed and the accompanying total mass-to-energy conversion? The answer: It is the only way to solve the several well-known paradoxes associated with black holes - particularly with minimal-mass black holes. As will be shown, reification of the lightspeed environment is essential to the physics of Terminal neutron stars.

It so happens that under certain conditions mass particles can, and do, quite literally fall into a state involving the ultimate speed. It is during the process of gravitational collapse that mass will, if the collapse involves a sufficient quantity of matter, become subjected to a lightspeed situation.

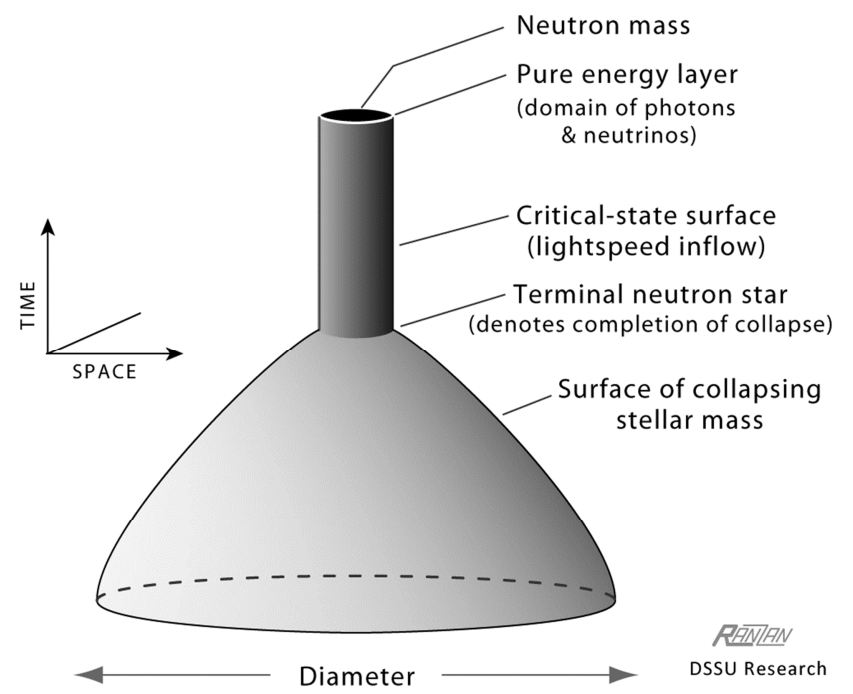

Figure 6. Gravitational collapse scenario. Natural end-stage collapse results in a Terminal neutron star. It has maximal gravity intensity; not even light can escape its surface. In terms of the aether theory of gravity, the universal space medium is flowing inward at the surface with a speed equal to that of light. Consequently, the structure has a thin layer consisting of pure energy in which for obvious reasons, only photons and neutrinos can exist. These energy particles are the remains of the mass that was compelled, by virtue of its radial location within the anatomy of the collapse, to travel at lightspeed (with respect to the inflowing aether).

Consider a star with a mass equivalent to about 3.4 times that of our Sun. It is at the end of its normal lifespan and it has no rotation. Imagine this massive star undergoing a simplified gravitational collapse. No sudden implosion, no rebound ejection, no nova event, and no supernova explosion - just a 
gradual contraction. See Figure 6. The gravitational contraction, in accordance with the unified theory of gravity [6], comes to an end with the formation of a Terminal-state neutron star.

A Terminal neutron star is a structure that cannot collapse further, cannot increase in density, and cannot increase in total contiguous mass. A fundamental self-regulating process imposes those features and makes the structure a true end-state star. But the most remarkable feature is its pure energy surface - a layer with trapped radiant energy. Here the universal space medium penetrates the surface traveling at the ultimate speed; it flows inward at the speed of light. Naturally, only photons and neutrinos can exist at, or in, such a surface. They travel at lightspeed with respect to the inflowing aether, while remaining stationary with respect to the neutron star.

Now notice what has happened in the process of gravitational collapse. The mass -including the main focus of the discussion, the electron - at the surface of the pre-collapsed structure quickly finds itself brought to a halt within a lightspeed environment. In such an environment, the mass particles have no choice, they must transform into their pure photonic state.

Trapped within the collapsed star's energy layer, a photon cannot deviate from a radial direction; it cannot exist as a loop; an electron's photon cannot even trace a helical path. Linear propagation is the reality here. Similarly other mass particles transform into linearly propagating photons and possibly neutrinos as well.

Thus, by removing the closed-loop condition and recognizing the open-helix nature, a door opens to a remarkably simple mechanism of total mass-to-energy conversion. Various related aspects of the mechanism are currently under investigation. Suffice to say, the implications for astrophysics are staggering.

\subsection{Historical Note}

There does not seem to be anything inherent in the electron's electromagnetic property that demands (or causes) a self-contraction.

It is interesting to note, although Albert Einstein endorsed the concept of electron contraction as originally advanced by H. A. Lorentz, he made it clear that "This hypothesis ... is not justifiable by any electrodynamical facts." [7]

\subsection{Realistic Alternative}

Based on the above three compelling arguments, the realistic alternative is that the electron propagates as a photonic OPEN loop —as a helix. Figure 7 demonstrates, with a paper-strip model, how the closed loop can be modified to form an open-loop (double-turn) electron. The resulting open configuration retains the electron's defining features; and the photon retains the circular polarization that produces the electric charge.

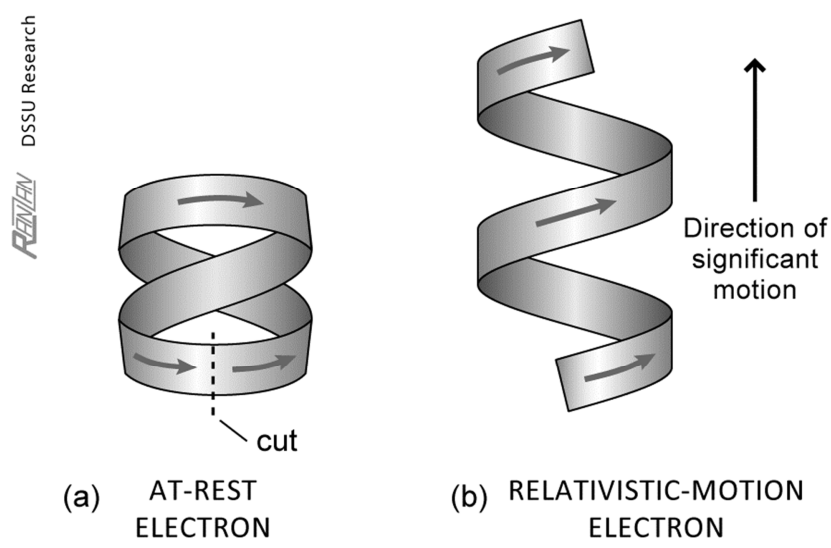

Figure 7. Closed-loop paper-strip model (a) is cut and stretched (b). The helical strip in (b) retains its length of one wavelength and represents an electron with significant linear motion. The photon does not have to double back, does not have to return to some arbitrary starting point, does not have to link up with its tail-end. Only the essential feature (the feature that defines the process as an electron) needs to be retained. The propagating photon just needs to be appropriately polarized.

The only way out, the only way to overcome the three objections, is to postulate that the relativistic electron travels as a helix, that is, the constituent photon propagates in helical fashion with no looping back in a retro direction.

The electron does not contract —it elongates!

\section{Electrons Apparently Stationary but Intrinsically Propagating}

Consider an elongated electron. Its constituent photon (propagating in helical fashion) has a reduced degree of confinement. The greater the speed of the electron, the lesser is the confinement. This means, in keeping with the definition, that the greater the speed of the electron, the smaller is its property of mass. The electron lowers its mass energy. This fundamental connection between mass and motion is supported by the research of H. I. Epstein in his article, Could Mass Decrease With Velocity?[8] As one would expect, a reduction in the mass energy also means there is a corresponding increase in kinetic energy. (This is graphically shown in Figure A2 of the Appendix.)

All of this raises an obvious question. What mechanism apportions the electrons mechanical energy between mass and motion? What determines the electron's mass energy as opposed to its kinetic energy?

The most rewarding way to examine the nature of electron elongation, and address the question of energy apportionment, is to consider the surface environment of extraordinarily dense compact stars.

As a concrete example, take a white-dwarf star with a mass equivalent to 1.4 Suns. (The white-dwarf type of star is ideal for the purpose here, as it is saturated with free electrons, electrons that have been stripped from their nuclei. These electrons, because they are so tightly packed, resist the gravitational collapsing to even greater density by what is called electron degeneracy pressure.) If its density is $10^{11}$ 
kilograms per cubic meter (and assumed uniform), then Newtonian equations give the star's radius as 1880 kilometers. And, in accordance with the aether theory of gravity, the inflow of aether into the structure must be approximately 14,000 kilometers per second. This is the inflow at the surface of the white-dwarf, as shown in Figure 8. From the diagram, it is easy to see that the electrons embedded in or near the surface are stationary with respect to the star; but they are propagating with respect to the aether. Surface electrons possess intrinsic motion of 14,000 kilometers per second.

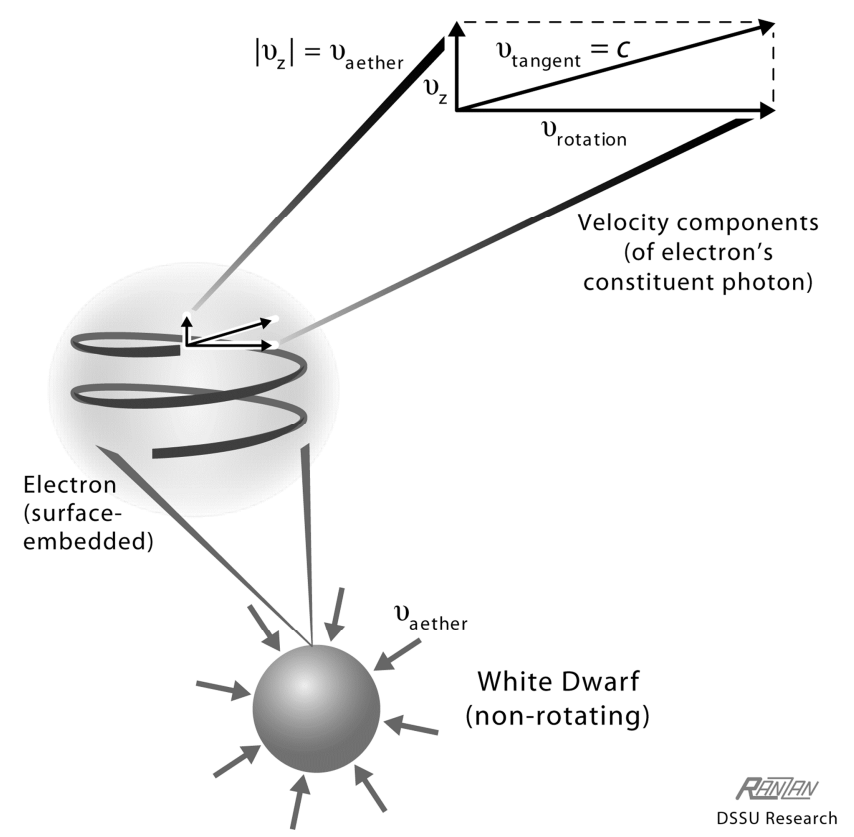

Figure 8. How a seemingly motionless electron manifests itself when trapped within an extreme gravitational environment. Shown is an electron apparently motionless (at-rest on the surface of a white-dwarf star) but, at the same time, intrinsically propagating (speeding through the space medium). Note the $z$-direction elongation of the electron. And note the constituent photon's velocity components: Vector $v_{\text {tangent }}$ is always tangent to the helical path; the $z$-axis component $v_{z} i$ s related to the electron's intrinsic kinetic energy; and the rotational component $v_{\text {rotation }}$ is related to the electron's intrinsic mass energy. (If the white-dwarf star has a mass of 1.4 Suns and a density of $10^{l 1} \mathrm{~kg} / \mathrm{m}^{3}$; then its radius will be 1880 kilometers and its aether inflow at the surface will be $14000 \mathrm{~km} / \mathrm{s}$.).

What sounds like a paradox is explained as the difference of the apparent absence of motion, on the one hand, and, on the other, the unseen underlying reality of the relative motion between the electrons and the subquantum medium.

As shown in Figure 8, the motion of the constituent photon is along a helical path. The motion's vector representation, labelled $v_{\text {tangent }}$, lies tangential to the helix and has a magnitude equal to the speed of light $c$. This vector has two components. One is in the vertical direction and is labelled $v_{z}$ and has a magnitude equal to the aether inflow. It is the component that determines the electron's intrinsic kinetic energy. The other is the rotation component (labelled $v_{\text {rotation }}$ ) and is responsible for the electron's intrinsic mass energy. The details for calculating these energies are provided in the Appendix. Needless to say, the vector sum of the two components must equal the speed of light $\left(v_{z}+v_{\text {rotation }}=v_{\text {tangent }}=c\right)$.
The white-dwarf's surface inflow of $14,000 \mathrm{~km} / \mathrm{s}$ seems extreme but it is not; it is only 5 percent of the speed of light and produces a corresponding 5 percent elongation of the electrons. For the truly extreme situations, it is necessary to turn to stars of much greater density - the neutron stars.

Imagine the same 1.4-Solar-mass Dwarf as it progressively acquires additional mass and transitions to the neutron-density state. Let it continue to acquire mass, enough mass to bring the surface environment to its ultimate extreme - at which the aether inflow attains the full speed of light. Figure 9 shows the electron elongation at various intervals of such a transition of a white dwarf into a Terminal neutron star. The progressive elongation is the mechanism by which the electron's confined photon becomes a free photon; the mechanism by which the photon's helical path transitions to a linear path; the mechanism by which mass energy is converted to radiant energy.

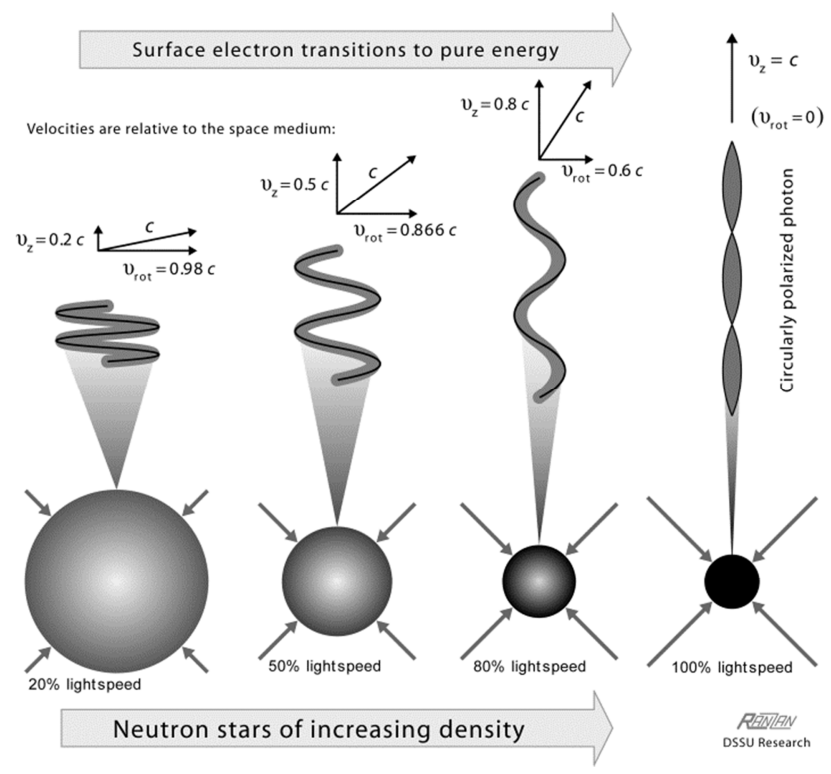

Figure 9. Surface-embedded electrons are elongated when subjected to extreme gravitational situations. For the electrons to remain stationary at the surface, they must race through the aether "headwind" at the various fractions of lightspeed as shown. Again, the $v_{\text {rot }}$ component vector determines the electron's intrinsic mass (which, therefore, varies with the photon's self-orbiting speed); and the vertical component vector represents the radial aether-referenced motion. At the surface of the Terminal neutron star, electrons no longer exist but have transformed into their constituent photons.

As mentioned earlier, it is important to realize that since no force has been applied to the electron, there should not be any change in the wavelength, not during the photon's helical propagation, and not during the final transition to linear propagation.

The surface electrons reach their maximum elongation (and complete their transformation to free photons) when gravity intensity becomes maximal, when the aether inflow rises to the full speed of light. Edward Harrison, in his popular textbook Cosmology the Science of the Universe, described the extreme environment as follows: "[The] effect is the same as if space itself were flowing ... inward at the speed of light. Lightrays moving outward at the ... surface remain at the 
same place; they move locally at the speed of light and travel through space that is itself falling in at the speed of light." [9]

What started out as stationary electrons in the "proper" frame of the neutron star's surface ended up as photons — photons propagating at lightspeed but going nowhere.

\section{Electron Loop \& Atomic Length Contraction}

None of what has been revealed about the electron, above, changes the phenomenon of length contraction as it relates to atoms and ordinary objects. Amazingly, the elongation behavior of the electron does not affect the opposite behavior - the contraction effect - of the atomic structure.

What is to be clarified in this section is the fundamental reason why - under conditions of significant motionelectrons (and probably also protons and neutrons) elongate, while atoms contract.

Within any given atom, electrons move about the nucleus in an orderly arrangement of orbitals. The path that an electron takes depends on the particular orbital, which, in turn, depends on its energy level. There are several possible path configurations; some simplified versions are shows in Figure 10. For any electron orbital (path), the absolutely essential feature is the circumnavigation of the atom's nucleus.
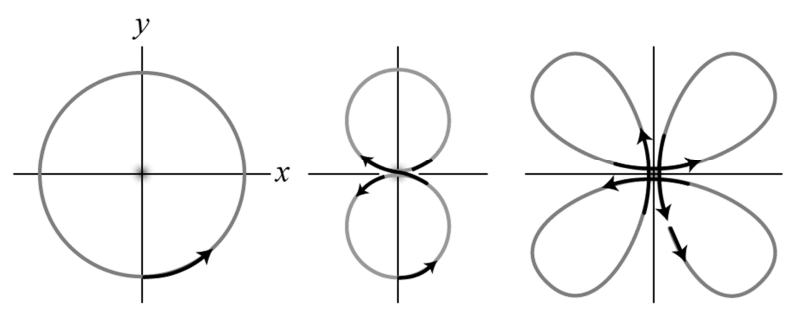

Figure 10. Electron orbitals about a central nucleus. Simplified paths of what are actually three-dimensional configurations.

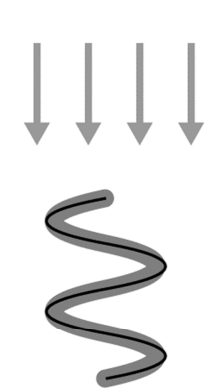

Aether flow

(a) Electron

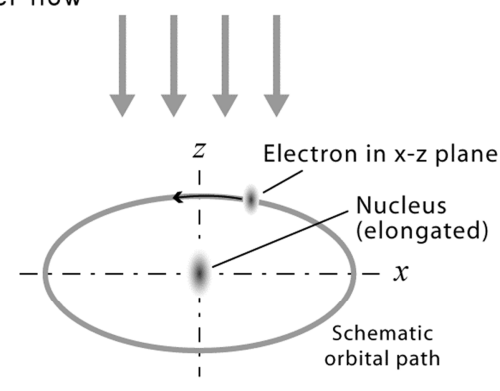

(b)

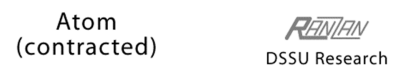

Figure 11. A key difference between the electron and the atom pertains to the photon loop versus electron loop. In the case of the electron (a), there is no central particle that causes the confinement - no central entity for the looping photon to orbit. However, the relationship is different with the atom (b); there the moving component, the electron, is confined by a central particle (the nucleus). The path of the electron must cyclically double back around the atom's nucleus. The result is that the electron elongates while the atom contracts. (Since protons and neutrons are also configurations of confined photons, the nucleus is shown elongated.).
Regardless of the speed of the atom, if the electron happens to be ahead at one instant, it must always double back and revisit the trailing side of the nucleus. Atoms have a particulate center; electrons do not.

Since the electron itself has no central particle, its constituent photon is not compelled to loop in a retrograde direction. See Figure 11.

The electron is a confined electromagnetic vortex. It consists of a photon that is self-confined. Although it is not known exactly what drives this confinement, the existence of a central particle as the cause can certainly be ruled out. However, in the case of the atom, there is a central mass that confines its orbiting leptons within its electromagnetic-field. A nuclear mass acting as the center of attractive force confines the electron cloud.

In the proper frame, i.e., in the frame of the atom, the path of the orbiting electron must be a closed path. Thus, in accordance with the closed-loop argument used earlier in Section 2, the path must contract along the direction of motion. The atom undergoes physical contraction [10].

Now here is a really important point. An atom that length-contracts does not increase in mass - it only increases in energy density. On the other hand, if the electron and the nucleons were to length-contract, there would necessarily be a mass increase (as explained in Section 3.1). So, on the basis of maintaining consistency with energy conservation, it is fitting that atoms and molecules undergo contraction while the fundamental particles themselves do not.

There is a new twist in the phenomenon of intrinsic length contraction. Remarkably, while subatomic particles (distinct in their photonic configuration patterns) undergo elongation, the atomic structures they constitute and define undergo contraction - a shrinkage along the dimension aligned with the direction of aether-referenced motion.

The electron itself elongates while the electron's orbital path contracts.

\section{Concluding Remarks}

The mystery behind confinement. As mentioned earlier, it is not known what maintains the photon's state of confinement. Gravity alone cannot sustain the confinement. Scottish physicist J. G. Williamson addresses this problem in the article "On the Nature of the Electron and Other Particles."[11] The mystery is compounded by the internal electric field that must surely be present. The question is, if the positive electric field lines-of-force are all directed inward, why isn't there a localized internal repulsion effect to oppose the confinement?

In any case, just as it is not known what restricts the photon to a closed loop in the at-rest electron, it is not known what restricts the same photon to a helical path when the electron undergoes significant motion.

Regarding conventional relativity. Nothing herein alters the predictions of conventional relativity theory. When relative motion is involved (relative motion between observer or his measuring instruments and the particles) length contraction will be an apparent phenomenon and will still be 
experimentally measurable. The electron as measured by its electric field will appear contracted when there is relative motion. It is only in the electron's proper frame during its relative-to-aether motion that elongation occurs. But just as physical length contraction of an object cannot be observed in the object's proper frame, elongation would not be observable in the electron's proper frame [10]. If one attempts to measure the electron's elongation by comparing it to an appropriately-sized measuring rod, say another electron, the measuring rod will elongate just as much as the target electron. No elongation is detected.

The paradox of unrestricted length contraction. This is the paradox within conventional relativity theory in which one may predict that a particle loses its volume (due to extreme relative motion) and also claim that, under the same circumstances, it retains a volume (within which the particle's energy resides).

Before explaining how the new understanding of contraction/elongation solves the paradox, it may be helpful to put this into the historical context. The situation of a particle with energy but with NO volume arises as follows. The faster an object or particle moves, the shorter it becomes. A particle moving with the velocity of light, it was long-believed, would lose its third dimension. It would become a cross-section of itself. The argument was that a mass particle, traveling at the speed of light, would become a mere cross-section of itself. Quoting Russian mathematician and philosopher P. D. Ouspensky,

"Einstein affirms that a rigid rod moving in the direction of its length is shorter than the same rod when it is in a state of rest, and the more quickly such a rod moves, the shorter it becomes. A rod moving with the velocity of light would lose its third dimension. It would become a cross-section of itself." [12]

Ouspensky added, "Lorentz himself affirmed that an electron actually disappears when moving with the velocity of light." [12]

So if Einstein is correct about the speeding rod and Lorentz is correct about the lightspeed/relativistic electron; then it seems logical to assume that, in the process of transforming into mere cross-sections, either the masses have vanished OR the masses exist in a state of zero volume! Stated more broadly, either the mass energy has vanished OR the mass energy exists within a zero volume.

However, another expert on relativity argues that the third dimension is not lost. The eminent physicist John A. Wheeler says that in the "extreme relativistic limit a particle of rest mass $m$ behaves — so far as concerns the laws of conservation of momentum and energy - in practically the same way as a photon." [5] A photon has a wavelength - which, therefore, means the original particle hasn't lost its third dimension after all. So which is it? Does the particle lose its third dimension or not? Since it has not lost its energy, one is faced with an internal contradiction in the theory.

Thus the paradox: the rules of relativity predict a total loss of length while also predicting the opposite - a length associated with the wavelength of a photon.
The new understanding resolves the paradox by recognizing that atoms and objects are subject to contraction, but their constituent fundamental particles of mass (electrons, protons, neutrons) are not.

The infinity problem in special relativity. A particle or object gains kinetic energy (and therefore increases its total energy) with the application of a force driving it to ever higher speed. The higher the speed, the greater is the total mechanical energy. According to the basic equations of special relativity, as a particle or object approaches lightspeed, its energy rises without limit. Wheeler and Taylor, experts on spacetime physics, describe an extreme theoretical example: If an ordinary hydrogen atom somehow attains a speed close to lightspeed - so that the gamma factor in the energy equation is equal to $7.1 \times 10^{13}$; then this single atom will possess the energy equivalence of a motorcycle cruising at 25 miles per hour [13]. As unrealistic as this sounds - and is; it is taken seriously by practitioners of standard physics. Supposedly, there is no upper limit to the energy a particle/object can manifest.

The infinity problem also infests the other motion-energy equation - the relativistic momentum equation. When a theory's predictions are clearly unrealistic (a proton with the energy of a coasting 170 kilogram motorcycle!) and lead to infinities, they signal that something is wrong with the interpretation.

So why do the equations go to infinity? What exactly, in the interpretation, is responsible? ... It is simply that the mass is treated as a constant. It is the official interpretation. "In the modern language of relativity theory there is only one mass, the Newtonian mass $m$, which does not change with velocity." [14]

In the equation for total energy, $E=\gamma m c^{2}$; and in the equation for kinetic energy, $K=m c^{2}(\gamma-1)$; and in the equation for momentum, $\mathbf{p}=\gamma m \mathbf{v}$;

the mass $\mathrm{m}$ is assumed to always remain constant (regardless of speed); while the speed-dependent gamma $(\gamma)$ factor has no theoretical upper limit.

This pitfall of infinite momentum and energy is entirely avoidable by simply recognizing that mass decreases with increase in motion. And the mechanism by which mass decreases is none other than fundamental particle elongation. Another important aspect is to appreciate the difference between apparent and intrinsic motions - the difference between observer-dependent motion and aether-referenced motion.

It also helps to recognize that the energy applied to a particle need not be the same as the energy "carried" by the particle. In other words, an unlimited amount of energy may be expended in an attempt to "push" a particle toward the speed of light without ever attaining that ultimate speed. (Yet by simply changing the gravitational environment, as was demonstrated earlier, any mass can be brought up to the ultimate speed, in the process of which it undergoes total mass-to-energy conversion. And here again, one must keep in mind the difference between apparent and intrinsic motions 
- the relative versus the aether-referenced.)

The paradox of stellar black holes. This paradox is based on the unrealistic extrapolation of general relativity. No doubt the easiest way to express this conundrum is with the question, How can there be mass/matter of infinite density inside a singular point of zero volume? And of course, the answer is that such a state is not possible, at least not in the real world. The red-flag of "infinity" is the only clue one needs. It is the clear warning that standard black-hole physics is fundamentally flawed. Nevertheless, there are physicists who seriously believe that the impossible (the existence of singular-type black holes) is possible. There are two main reasons why they believe: (Naturally, we all share the same evidence, so that in itself does not provide a reason.) Firstly, they have mathematical proof that the total gravitational collapse of a sufficient quantity of matter must end with all the gravitating mass inside a dimensionless speck - and still continue to gravitate. Dedication to the Platonic view may well be a prerequisite; the mathematics is held to be more real than the objects and the actions that the numbers represent. Secondly, they believe it because they have failed to find a mechanism with the power to preclude the collapse to singularity.

The new understanding (with its reinterpretation of the energy triangle as shown in the Appendix) resolves the paradox by facilitating the total conversion of mass to energy -without having to apply energy to the mass in the conventional manner. This turns out to be the key element in the remarkable mechanism [15] that precludes the formation of singularity-type black holes. Not only does the new interpretation banish the singularity concept from the physics of total gravitational collapse, but it also provides the driving energy behind astrophysical jets [15] [16]. Such are the benefit to physics of incorporating the concept of particle elongation.

As for the misguided belief in the primacy of mathematics: the real world is ruled by processes, not by numbers, not by equations.

The traditional view versus the new interpretation.

Where, within the long-established conviction, does the energy go if the particle, atom, or object becomes a cross-section of itself? Where does the energy go when the thing has no volume, when it exists only as a cross-sectional area and no thickness dimension? Where indeed! Essentially, under the traditional view, one cannot combine unrestricted length contraction and energy conservation —not in a realistic way. The two are mutually exclusive concepts. And for this reason the long-standing rule has been that a mass particle/object can never attain lightspeed. And that rule, in turn, has been most responsible for the failure to understand the nature of the strangest objects of the Cosmos - Terminal neutron stars.

The opposing view is that length contraction is conditional; it affects atoms and objects, but not elementary particles. Mass still cannot attain lightspeed; however, this new interpretation of motion gives mass the ability to convert totally to energy. Given the appropriate gravitational circumstances mass can, and does, undergo complete conversion to radiant energy
- photonic energy trapped in the surface of a Terminal neutron star and available for feeding its emission beams and driving external jets.

The new interpretation centers on the aether-referenced elongation of fundamental particles and provides a remarkable fit to the reality. The specific benefits of this concept include: (i) It facilitates the conservation of intrinsic energy. (ii) It makes possible the decrease of mass with increased motion (with respect to aether). (iii) It provides the key element in the mechanism of mass-to-energy conversion - a noninteraction-hundred-percent conversion (i.e., no particle-antiparticle annihilations is involved). (iv) It precludes the formation of singularity-type black holes.

Something unexpected, something unambiguously compelling. After many years (since 2001) of predictive success of the Cellular Universe model and DSSU theory, there emerged an unexpected yet necessary phenomenon - the elongation of fundamental particles involved in aether-referenced motion.

Theoretical physicist Lee Smolin has an interesting warning in his book The Trouble With Physics. He says that the theories massaged and framed from the data - what one would call the triumphs of human thought - are always circumspect. At least, they should be. "Science moves forward when we are forced to agree with something unexpected."

\section{Appendix}

\section{Relativistic Apparent-Energy Triangle}

A most useful, and easy-to-understand, form of the mechanical energy relationship is

$$
\left(\begin{array}{c}
\text { Total } \\
\text { energy }
\end{array}\right)^{2}=\left(\begin{array}{c}
\text { Rest } \\
\text { energy }
\end{array}\right)^{2}+\left(\begin{array}{c}
\text { Momentum } \\
\text { energy }
\end{array}\right)^{2}
$$

Notice that kinetic energy is not explicitly stated; but be aware that it is part of the "Total energy" expression on the left side of the equation. The next equation clarifies this point.

Using the terminology shown in Figure Ala, the Total energy may be expressed as

$$
E=E_{0}+E_{\text {kin }}=\sqrt{\left(m c^{2}\right)^{2}+(\mathbf{p} c)^{2}} .
$$

The special relativity interpretation of mechanical energy: A crucial factor in the special relativity view is that mass is treated as having constant value. The rest mass of a particle or object does not change with its speed. The problem is that such an assumption permits the acquisition of unlimited mechanical energy. Part (b) of Figure A1 demonstrates the energy triangle's prediction of limitless energy. As the motion approaches lightspeed, the energy tends to infinity. The triangle rises without limit as the mass supposedly gains ever more energy.

No one can fail to see that this textbook interpretation precludes mass-to-energy conversion in association with motion. 


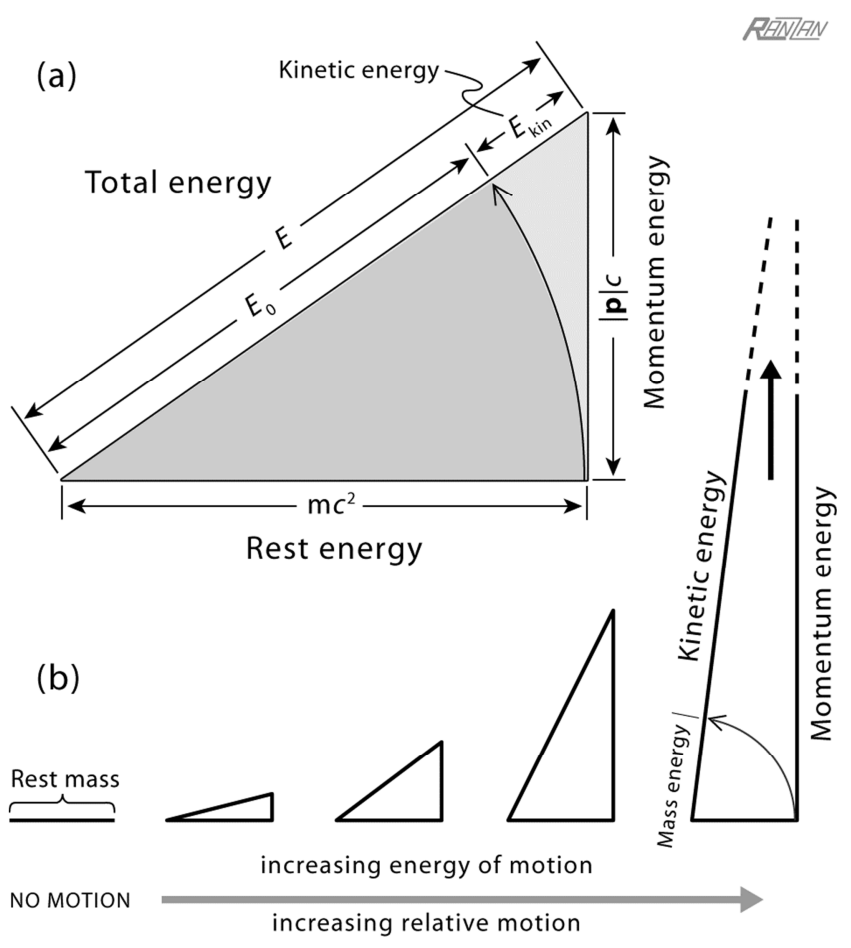

Figure A1. Special relativity interpretation of the energy relationship as represented by the triangle in (a). The energy triangle embodies the Pythagorean relationship among the energy components associated with bodies or particles. The two crucial aspects of the interpretation, as demonstrated in the sequence (b), are: (i) mass remains constant irrespective of motion; (ii) kinetic and momentum energies increase with greater motion, and may do so without theoretical limit. (Note the lack of symmetry here - the triangle collapses at one end of the sequence but not at the other end- then compare this with the sequence in Figure A2.).

\section{Aether-Referenced Energy Triangle}

Under the new interpretation the velocities of particles or objects are referenced to the universal space medium - the aether. Consequently, all the usual parameters acquire what may best be called intrinsic status; that is, they become qualitatively and quantitatively tied to the aether medium. (It does not mean there is some intrinsically fixed value involved. The various energies are subject to the intrinsic motion with respect to aether, rather than being relative to some observer or some moving frame.)

According to the intrinsic interpretation, mass is treated as a function of aether-referenced motion. Expressed in terms of the intrinsic gamma factor,

$$
m_{\text {int }}=\frac{m_{0}}{\gamma_{\text {int }}} .
$$

And in terms of the intrinsic velocity,

$$
m_{\mathrm{int}}=\sqrt{1-\left(v_{\mathrm{int}} / c\right)} m_{0} .
$$

As convention has it, $m_{0}$ is the rest mass and remains constant within the equation (i.e., $m_{0}$ is not a variable parameter).
Notice the permissible range. The mass can now vary from $m_{0}$, when $v_{\text {int }}$ is negligible, down to zero, when $v_{\text {int }}$ is equal to the speed of light. It can vary from rest-mass-value $m_{0}$ all the way down to zero as shown in Figure A2b.

The form of the standard equations for energy and momentum is retained. With suitable subscripts, equation (A2) is still useful. Only the mass term $m$ must be replaced by $m_{\text {int }}$; and the velocity parameter must be replaced by $\gamma_{\text {int }}$ or $v_{\text {int }}$.

And again, the mechanical energy relationship is represented by a right angled triangle (Figure A2a).
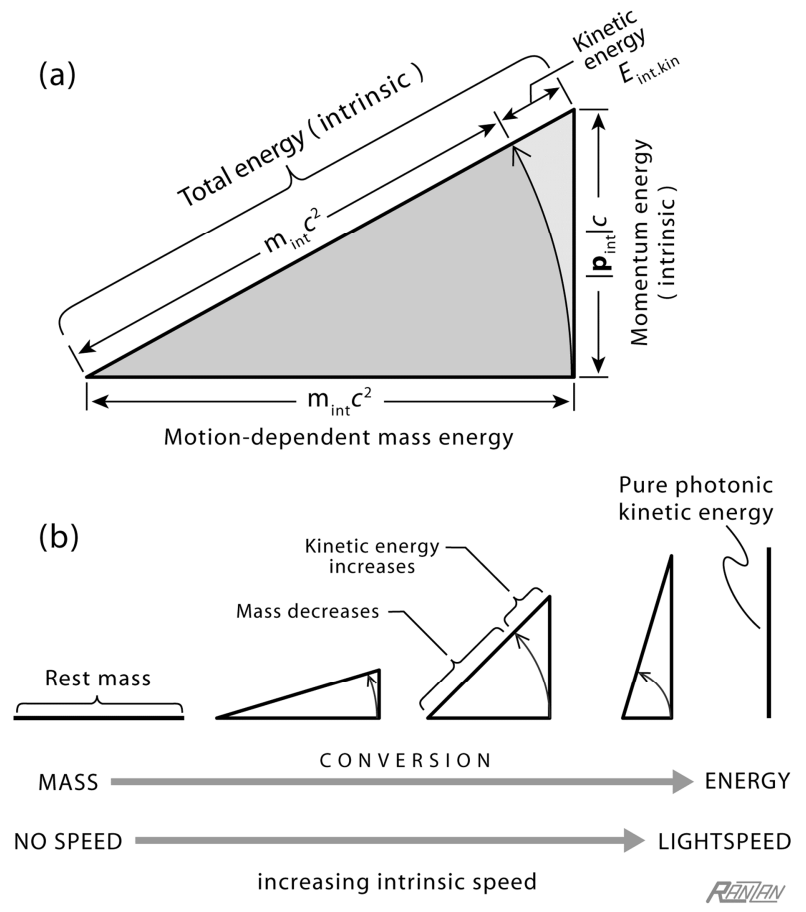

Figure A2. Manipulation of the energy triangle under the condition of substrate-referenced motion. (a) Energy triangle applicable to particles/objects for which velocity is referenced to the universal space medium. Intrinsic mass $m_{\text {int }}$ is defined as $\left(m_{0} / \gamma_{\text {int }}\right)$. And momentum $\boldsymbol{p}_{\text {int }}$ is defined as $\left(m_{\text {int }} \cdot v_{\text {int }}\right)$. (b) Schematic demonstration of mass-to-energy conversion. (The hypotenuse is held constant as the base shrinks with increasing speed). Under this interpretation the total energy remains constant; it is recognized that the energy expended in accelerating a particle is not necessarily equal to the energy it retains. Most importantly the "intrinsic" interpretation models the situation in which nominally stationary objects undergo extreme aether-referenced motion -namely, the gravitational situation in which mass undergoes 100-percent conversion to radiant energy.

There are two obvious advantages of this new interpretation. One is the conservation of total energy (no off-the-chart extrapolation). The other is in the simple way by which mass-to-energy conversion can be explained. As explained elsewhere [17], such total conversion occurs during and following the formation of Terminal neutron stars; and requires the phenomenon of fundamental particle elongation.

\section{References}

[1] Internet article: Electron, https://en.wikipedia.org/wiki/Electron (2018-12-26). 
[2] Qiu-Hong Hu. (2004) The Nature of the Electron, Physics Essays Vol.17, No.4, pp. 442-458 doi: http://dx.doi.org/10.4006/1.3025708.

[3] Williamson, J. G. and van der Mark, M. B. (1997) Is the Electron a Photon With Toroidal Topology? Annales de la Fondation Louis de Broglie, Vol.22, No.2, 133.

[4] Okun, L. B. (1989) The Concept of Mass, Physics Today, V.42, No.6, 31. (http://dx.doi.org/10.1063/1.881171 ).

[5] Wheeler, J. A. and Taylor, E. F. (1966) Chap 2. in Spacetime Physics. W. H. Freeman, San Francisco. P121.

[6] Ranzan, C. (2018) The Nature of Gravity -How one factor unifies gravity's convergent, divergent, vortex, and wave effects, International Journal of Astrophysics and Space Science (IJASS), Vol.6, No.5, pp.73-92. (Doi: http://dx.doi.org/10.11648/j.ijass.20180605.11).

[7] Einstein, A. (1952) Relativity The Special and the General Theory. Wings Books, New York, 1961 edition. P57.

[8] Epstein, H. I. (2009) Could Mass Decrease With Velocity? Physics Essays, Vol.22, No.4, pp511-516. (http://dx.doi.org/10.4006/1.3227028)

[9] Harrison, E. R. (1981) Cosmology the Science of the Universe. Cambridge University Press, U. K. P191.

[10] Ranzan C. (2013) The Physical Nature of Length Contraction, Applied Physics Research, Vol.5, No.1. (Doi: 10.5539/apr.v5n1p87)
[11] Williamson, J. G. (2008) On the nature of the electron and other particles, Presentation at The Cybernetics Society 40th Anniversary Annual Conference (2008 Sept 20) in London. (www.cybsoc.org/cybcon2008prog.htm).

[12] Ouspensky, P. D. (1931) A New Model of the Universe (originally published by Alfred A. Knopf, Inc., 1931. (Vintage Books edition, N. Y., 1971) P360.

[13] Wheeler, J. A. and Taylor, E. F. (1966) Chap 2. in Spacetime Physics. W. H. Freeman, San Francisco. P116.

[14] Okun, L. B. (1989) The Concept of Mass, Physics Today, V.42, No.6, 31. (http://dx.doi.org/10.1063/1.881171 ) P31.

[15] Ranzan, C. (2018) Natural Mechanism for the Generation and Emission of Extreme Energy Particles, Physics Essays, Vol.31, No.3, pp.358-376. (Doi: http://dx.doi.org/10.4006/0836-1398-31.3.358).

[16] Ranzan, C. (2019) Nature's Supreme Mechanism for Energy Extraction from Nonmaterial Aether, Infinite Energy, Vol.25, Issue\#144.

(http://www.infinite-energy.com/iemagazine/issue144/index.ht $\mathrm{ml})$.

[17] Ranzan, C. (2019) Mass-to-Energy Conversion, the Astrophysical Mechanism, Journal of High Energy Physics, Gravitation and Cosmology (JHEPGC) Vol.5, No.2, pp.520-551. (Doi: https://doi.org 10.4236/jhepgc.2019.52030). 